\title{
Impacto da perda dentária na qualidade de vida relacionada a saúde bucal de adultos
}

\author{
Impact of tooth loss on oral health-related quality of life of adults
}

\author{
Johelle de Santana Passos-Soares ${ }^{1 *}$, Isaac Suzart Gomes-Filho ${ }^{2}$, Lilia Paula Souza Santos ${ }^{3}$, \\ Pedro Nascimento Prates Santos ${ }^{4}$, Ivana Conceição Oliveira da Silva ${ }^{3}$, \\ Izadora da Silva Campodonio Eloy Balinha ${ }^{3}$, Soraya Castro Trindade ${ }^{5}$
}

\begin{abstract}
${ }^{1}$ Professora Adjunta da Faculdade de Odontologia da Universidade Federal da Bahia(UFBA); ${ }^{2}$ Professor Pleno da Universidade Estadual de Feira de Santana (UEFS); ${ }^{3}$ Mestre em Saúde Coletiva pela UEFS; ${ }^{4}$ Professor Assistente da UEFS; ${ }^{5}$ Professora Titular da UEFS.
\end{abstract}

\begin{abstract}
Resumo
Introdução: a perda dentária parece impor alterações físicas, biológicas e psíquicas que podem impactar negativamente na qualidade de vida dos indivíduos. Objetivo: avaliar a associação entre perda dentária e a percepção do seu impacto negativo na qualidade de vida. Metodologia: estudo transversal, conduzido com 306 adultos, atendidos em serviços públicos de saúde, em Feira de SantanaBa. O impacto na qualidade de vida relacionada à saúde bucal foi mensurado pelo OHIP-14. Foi realizado exame bucal e aplicado questionário estruturado para obter informações relacionadas às características socioeconômicas, demográficas, de estilo de vida e condições de saúde geral e bucal. Foi empregado o Teste qui-quadrado e estimadas as Razões de prevalência e intervalos de confiança a 95\% (IC95\%). Resultados: dentre as dimensões do OHIP-14, a perda dentária ( $\geq 7$ dentes) foi estatisticamente associada com a dimensão "incapacidade física" (RP: 1,50; IC95\%: 1,18-1,90, p=0,003). Conclusão: houve associação entre alta perda dentária e impacto da saúde bucal na qualidade de vida, expressa pela incapacidade física.
\end{abstract}

Palavras-chave: Qualidade de vida. Perda de dente. Saúde bucal. Autoimagem.

\begin{abstract}
Introduction: tooth loss seems to impose physical, biological and psychological alterations which could impact negatively on quality of life of individuals. Objective: was to evaluate an association between tooth loss and oral health-related quality of life. This crosssectional study examined a sample of 306 adults, attended at public health services, in Feira de Santana-Ba. Metodology: the 14-item Oral Health Impact Profile (OHIP-14) was used to capture the impact on their quality of life. Oral examination was performed and a structured questionnaire was applied to obtain more information related to socioeconomic and demographic variables, lifestyle one and general and oral health conditions. The chi-square test was used and the prevalence ratios (PR) and confidence intervals at $95 \%$ (IC95\%) were estimated. Results: among the specific dimensions of the OHIP-14, tooth loss ( $\geq 7$ teeth) was statistically associated with the dimension "physical disability" (RP: 1.50; IC95\%: 1.18-1.90, p=0.003). Conclusion: there was association between high tooth loss and oral health-related quality of life, expressed by physical disability.

Keywords: Quality of life. Tooth loss. Oral health. Self concept.
\end{abstract}

\section{INTRODUÇÃO}

A carga global das condições bucais na população tem aumentado nas últimas décadas, revelando o impacto de agravos bucais e de suas consequências nos anos de vida saudáveis perdidos ou vividos com incapacidade. Estudos mostram que, no ano de 2010, um total de 3,9 bilhões de pessoas foram afetadas por problemas bucais como a periodontite grave e cárie dentária, principais doenças que quando não tratadas oportunamente incorrem em perdas dentárias ${ }^{1-3}$. A perda dentária tem se mostrado como a 36a condição de saúde bucal mais prevalente, com estimativa global de $2,3 \%$ na população, sendo evi-

Correspondente/Corresponding: *Johelle de Santana Passos Soares End: Av. Araújo Pinho, 62 - Canela CEP: 40110-150-Salvador (BA) - Tel: (71) 3283-1347/8964 -E-mail: johpassos@gmail.com denciada mais comumente entre os indivíduos com idade mínima de 65 anos e no sexo feminino ${ }^{1,3}$.

Dados estimados no Brasil indicam que o número de pessoas afetadas pela cárie tem diminuído, levando ao menor CPOD da América Latina. Porém, ressalta-se nesse quadro de saúde que $23 \%$ dos seus idosos são edêntulos totais e mais $24 \%$ necessitam de próteses parciais em uma das arcadas ${ }^{4}$. Esses valores são maiores que a média global de desdentados, e juntamente com o Brasil, entram para esse ranking a Turquia, Irã, Nova Zelândia e México, todos apresentando perda dentária grave ${ }^{1}$.

Essa grande demanda reprimida de adultos com acúmulo de necessidades odontológicas tem levado pesquisadores a investigar o impacto da condição bucal na qualidade de vida. A Organização Mundial de Saúde (OMS) coloca o conceito de qualidade de vida em um 
contexto amplo e complexo, determinado pela saúde física, estado psicológico, nível de independência, relações sociais, crenças pessoais do indivíduo e pelas condições de vida em que se vive. Assim, define qualidade de vida como a percepção que cada pessoa tem da sua posição na vida, dentro do contexto cultural e dos valores nos quais ele vive e em relação aos seus objetivos, expectativas e inquietações ${ }^{5}$.

Ao inserir a perda dentária nesse contexto de avaliação da qualidade de vida, percebe-se que seu impacto vai além da estética, causando transtornos funcionais e, principalmente, psicológicos ${ }^{6,7}$. Ressalta-se ainda que a rotina diária dessas pessoas também é alterada sofrendo modificações pela interferência na fala, alterações comportamentais, dificuldades de mastigação, no convívio social e na autoestima ${ }^{6}$.

Dessa forma, avaliações clínicas apenas não possibilitam mensurar o nível de saúde do indivíduo, requerendo análise das suas percepções sobre a sua condição bucal, de modo a auxiliar no refinamento diagnóstico e identificação de grupos populacionais vulneráveis ${ }^{8}$. 0 uso de instrumentos que avaliem a percepção do indivíduo sobre sua qualidade de vida relacionada a saúde bucal tem sido cada vez mais utilizado em levantamentos epidemiológicos, a exemplo do Oral Health Impact Profile (OHIP), que através de escores estabelecem o grau do impacto da saúde bucal na qualidade de vida dos indivíduos, levando em conta os aspectos físicos, sociais e psicológicos, ${ }^{8-10}$. A versão brasileira desse questionário validada e resumida, - OHIP-14, tem possibilitado identificar o domínio (físico, social ou psicológico) mais comprometido e assim, orientar os profissionais no melhor entendimento em relação à condição de saúde de seus pacientes, aos aspectos que parecem ser valorizados pelos mesmos e que impactam em suas vidas.

Nessa perspectiva, estudos de autopercepção da perda dentária podem contribuir para ajudar a entender quais as necessidades e fragilidades relacionadas, e pensar estratégias efetivas de controle de doenças da cavidade bucal, melhorando assim a qualidade de vida dessas pessoas.

Sendo assim, o presente trabalho se propõe a descrever a perda dentária em indivíduos adultos atendidos em serviços públicos de saúde de um município da Bahia - Brasil, relacionando esse agravo com o impacto negativo na qualidade de vida.

\section{METODOLOGIA}

\section{DESENHO DO ESTUDO E SELEÇÃO DA AMOSTRA}

Trata-se de um estudo epidemiológico do tipo corte transversal realizado em indivíduos atendidos nos serviços públicos de saúde em Feira de Santana-Ba, durante o período de Dezembro de 2015 a Fevereiro de 2016.
Esta pesquisa teve a aprovação do Comitê de Ética em Pesquisa da Universidade Estadual de Feira de Santana (CAAE: 47927115.8.0000.0053).

Os locais de estudo englobaram algumas Unidades de Saúde e o Centro de Atendimento ao Diabético e Hipertenso (CAHD) do município de Feira de Santana/BA. A escolha desses espaços foi por amostragem de conveniência de modo a assegurar a logística do estudo.

O cálculo amostral para esse estudo levou em consideração a frequência de $38,7 \%$ do impacto negativo na qualidade de vida entre os não expostos a maior perda dentária, Razão de prevalência de 1,31, poder de $80 \%$, nível de confiança de $95 \%$ e a proporção $1: 1$ entre os grupos de comparação. O número mínimo de indivíduos estimado para compor a amostra foi de 540 indivíduos.

A população foi composta por usuários com idade mínima de 18 anos presente nos serviços públicos de saúde. Não foram incluídos na pesquisa os indivíduos que tivessem realizado tratamento periodontal nos últimos três meses anteriores à pesquisa; apresentassem neoplasias; gestantes; ou incapacitados de compreender ou responder ao questionário.

\section{COLETA DE DADOS}

Após consentimento assinado dos participantes para inclusão na pesquisa, foram aplicados dois instrumentos de coleta estruturados para registro das informações e percepções: um questionário com perguntas sobre as condições socioeconômico-demográficas, estilo de vida, cuidados com a saúde e atenção odontológica; e o OHIP-14. Em seguida, em espaço reservado, sob luz natural e com uso de espelho bucal e sonda milimetrada tipo Willians (Hu-Friedy, Chicago, IL), foi realizado exame clínico bucal por Cirurgiã-Dentista previamente treinada.

O exame bucal consistiu de avaliação das medidas de profundidade de sondagem ${ }^{11}$, recessão/hiperplasia, nível de inserção clínica ${ }^{12}$, índice de placa ${ }^{13}$, sangramento gengiva $\left.\right|^{14}$ e registro dos dentes cariados, restaurados e extraídos através do emprego do índice de CPO-D, conforme preconizado pela Organização Mundial de Saúde ${ }^{5}$. $\mathrm{O}$ exame periodontal foi realizado em todos os dentes existentes em boca, exceto terceiros molares, e nos seis sítios: disto-vestibular, médio-vestibular, mésio-vestibular, disto-lingual, mésio-lingual e mésio-lingual.

A avaliação da reprodutibilidade foi feita através de medidas periodontais replicadas pelo examinador, tendo um periodontista experiente como referência, em cerca de $10 \%$ da amostra. Os coeficientes de correlação intraclasse alcançados para recessão/hiperplasia e profundidade de sondagem foram, respectivamente, 0,71 (IC95\%:0,25$0,91, p=0,004)$ e 0,91 (IC95\%:0,73-0,97; $p<0,001)$, sugerindo grau de concordância variável entre satisfatório e excelente. Para avaliação de cárie dentária, foi aplicado o teste Kappa obtendo-se o valor de 0,84 , considerado uma concordância dentro do grau excelente. 


\section{DETERMINAÇÃO DO IMPACTO NEGATIVO NA}

QUALIDADE DE VIDA

O impacto negativo na qualidade de vida foi medido pelo instrumento OHIP-14, na sua versão brasileira, que contém 14 itens ${ }^{15}$, agregados em sete dimensões: limitação funcional, dor física, desconforto psicológico, incapacidade física, incapacidade psicológica, incapacidade social e desvantagem social As perguntas foram respondidas em uma escala de Likert com os seguintes escores: nunca $=0$; quase nunca $=1$; às vezes $=2$; quase sempre $=3$; sempre $=4$. Os escores finais variam de 0 a 56 pontos, com escores maiores representando maior impacto na qualidade de vida.

Esse instrumento foi reaplicado em $10 \%$ dos indivíduos entrevistados dentro do intervalo de tempo de 01 semana, para avaliar a sua confiabilidade. A estabilidade e consistencia interna do instrumento OHIP foram avaliadas com emprego do coeficiente alpha-Cronbach, obtendo-se coeficiente geral alto $(\alpha=0,84)$.

Neste estudo, as pontuações foram descritas para a prevalência, extensão e gravidade do OHIP-14, de acordo com o sistema estabelecido por Slade et al. em 2005:

a. Prevalência: percentagem de indivíduos que responderam um ou mais itens com "quase sempre" ou "sempre";

b. Extensão: soma do número de itens com impacto, variando de 0 a 14, considerando as perguntas respondidas pelas opções "quase sempre" ou "sempre";

c. Gravidade: soma de todos os escores, variando de 0 a 56, sendo que quanto maior o escore, maior é o impacto decorrente das condições bucais sobre a qualidade de vida.

\section{ANÁLISE DE DADOS}

Foi feita uma análise descritiva de todas as covariáveis consideradas de interesse neste estudo segundo a perda dentária. Os pontos de corte utilizados para categorizar as covariáveis foram estabelecidos de acordo a sua distribuição na amostra ou com estudos prévios sobre o tema. A variável exposição (perda dentária) foi dicotomizada com base na sua mediana (sete). A variável dependente (impacto da condição bucal na qualidade de vida) foi avaliada segundo a sua distribuição percentual nos diferentes domínios do OHIP-14, bem como por sua prevalência, extensão e gravidade dos escores médios. Medidas de frequência simples e de tendência central foram obtidas. Análise bivariada foi realizada usando o teste Qui-Quadrado para variáveis categóricas e teste $t$ de Student para variáveis contínuas, com nível de significância de 5\%, para comparar as proporções e médias das covariáveis analisadas, respectivamente, entre os grupos com perda dentária $\leq 7$ ou $>7$. A magnitude das associações entre cada covariável e a variável independente foi calculada obtendo-se as Razões de prevalência (RP) e intervalos de confiança de 95\% (IC 95\%).

\section{RESULTADOS}

A amostra final obtida foi de 306 participantes, sendo divididos em dois grupos para comparação: grupo com perda dentária de até 7 dentes perdidos $(n=159)$ e grupo com mais de 7 dentes perdidos ( $n=149)$. A média de idade foi de 45 anos (desvio padrão= 14,8 anos), sendo a idade mínima de 18 anos e a máxima de 80 anos. A perda dentária de pelo menos uma unidade na amostra foi de 49,05\%.

Houve maior frequência de participantes do sexo feminino (73,5\%), com faixa etária de 45 a 80 anos de idade (51\%), cor de pele/etnia não branca $(92,2 \%)$, desempregado ou sem trabalhar $(55,7 \%)$, com nível de escolaridade até 8 anos de estudo $(57,2 \%)$ e recebendo pelo menos um salário mínimo $(75,4 \%)$.

Na Figura 1 são apresentados os dados da análise bivariada da associação entre perda dentária e fatores socioeconômicos-demográficos. Quando comparado aos indivíduos não expostos, a frequência de alta perda dentária ( $\geq 7$ dentes) foi maior entre os expostos com faixas etárias entre 35 a 44 anos $(30,9 \%, p<0,01)$ e 45 a 80 anos $(25,6 \%, p<0,01)$, entre desempregados $(54,1 \% p=0,03)$, com nível de escolaridade $\leq 8$ anos $(58,9 \%, p<0,01)$, que recebiam menos de 01 salário mínimo $(58,9 \%, p=0,02)$, e com número de filhos maior que $3(62,9 \%, p<0,01)$. Na Figura 2, as razões de prevalência obtidas mostram a força da associação entre essas variáveis e a alta perda dentária, e o não cruzamento da linha vertical expressa a significância estatística $(p<0,05)$. Pode se observar que as RPs estatisticamente significantes variaram entre 1,33 a 8,55 entre os grupos expostos mencionados acima. Dentre as medidas apresentadas, a maior magnitude da RP foi para idade entre 45 a 80 anos, indicando que a probabilidade de ter alta perda dentária é cerca de 8,6 vezes maior (RP: 8,55; IC95\%:3,96-18,47), do que nos indivíduos com entre 18 a 34 anos.

Figura 1 -Distribuição percentual das características socioeconômicas, demográficas e de estilo de vida segundo a perda dentária. Feira de Santana, Bahia, Brasil. $(n=306)$.

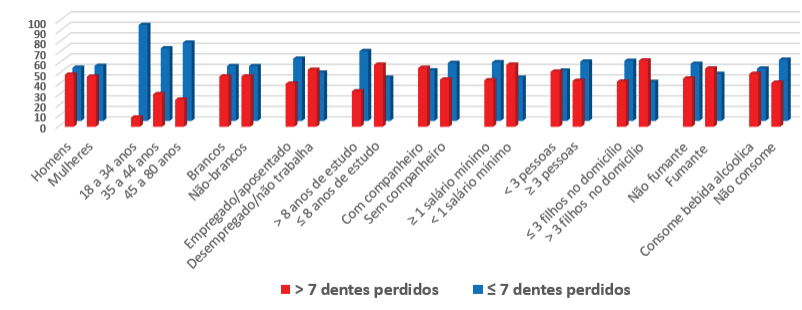

Fonte: Autoria própria 
Figura 2 - Razão de prevalência (RP) e intervalos de confiança a 95\% (IC95\%) da associação entre características socioeconômicas, demográficas e de estilo de vida e a perda dentária. Feira de Santana, Bahia, Brasil. $(n=306)$.

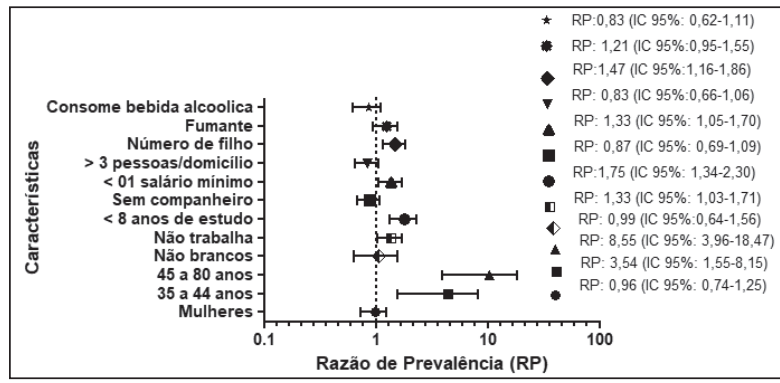

Fonte: Autoria própria

Quanto às condições e cuidados de saúde geral e bucal dos indivíduos (Figura 3), apenas hipertensão apresentou significância estatística. O percentual da alta perda dentária ( $\geq 7$ dentes) foi maior no grupo exposto à hipertensão do que entre os indivíduos sem hipertensão, sendo essa diferença estatisticamente significante $(p<0,01)$. A razão de prevalência obtida para esta associação expressou uma probabilidade 1,47 vezes maior de alta perda dentária na presença da hipertensão (RP: 1,47; IC95\%:1,15-1,86).

Figura 3 - Distribuição percentual das características relacionadas a condições e cuidados de saúde geral e bucal segundo a perda dentária. Feira de Santana, Bahia, Brasil. $(n=306)$.

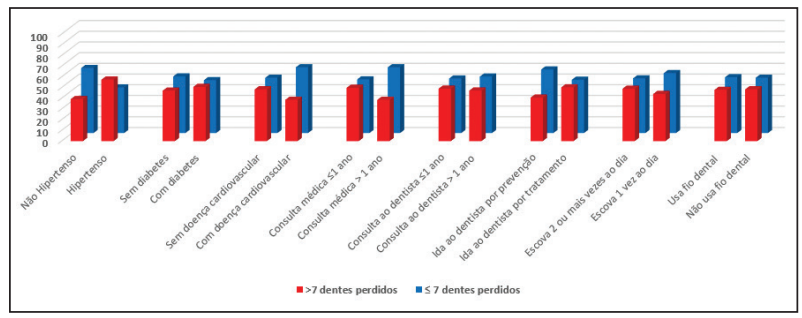

Fonte: Autoria própria

Quando avaliada a condição bucal dos participantes (Tabela 1), registrou-se, com significância estatística, entre os indivíduos com alta perda dentária, uma menor média de dentes restaurados $(p<0,01)$ e maiores valores médios de CPO-D $(p<0,01)$, índice de placa visível $(p=0,02)$ e nível de inserção clínica $(p<0,01)$.

Tabela 1 - Médias e desvio padrão de medidas clínicas bucais segundo perda dentária. Feira de Santana, Bahia, Brasil. (n=306).

\begin{tabular}{|c|c|c|c|}
\hline \multirow[t]{2}{*}{ MEDIDAS CLÍNICAS BUCAIS } & \multicolumn{3}{|c|}{ PERDA DENTÁRIA } \\
\hline & $\begin{array}{c}\leq 7 \\
(N=159)\end{array}$ & $\begin{array}{c}>7 \\
(N=147)\end{array}$ & $\mathrm{p}$ \\
\hline & Média $\pm D P$ & Média $\pm D P$ & \\
\hline Número de dentes restaurados & $5,75 \pm 4,85$ & $3,57 \pm 3,78$ & $<0,01$ \\
\hline Número de destes cariados & $2,85 \pm 3,41$ & $2,41 \pm 2,89$ & 0,23 \\
\hline Índice CPO-D (n) & $11,89 \pm 5,70$ & $20,45 \pm 4,65$ & $<0,01$ \\
\hline Índice de placa visível (\%) & $38,81 \pm 29,13$ & $47,30 \pm 31,95$ & 0,02 \\
\hline Sangramento à sondagem (\%) & $33,47 \pm 22,73$ & $37,74 \pm 24,39$ & 0,11 \\
\hline Profundidade de sondagem $(\mathrm{mm})$ & $2,40 \pm 0,62$ & $2,50 \pm 0,77$ & 0,25 \\
\hline Nível de inserção clínica (mm) & $2,70 \pm 0,91$ & $3,55 \pm 1,39$ & $<0,01$ \\
\hline
\end{tabular}

Rev. Ciênc. Méd. Biol., Salvador, v. 17, n. 2, p. 158-163, mai./jun. 2018
A prevalência do impacto da saúde bucal na qualidade de vida (respostas "quase sempre" ou "sempre" em pelo menos uma das 14 questões) foi de $60,5 \%$. A gravidade do impacto da saúde bucal, representada pelo somatório de escores obtidos no OHIP - 14, variou de zero a 46 , com média de $11,07 \pm 9,45$. Por sua vez, a extensão desse impacto, que expressa a quantidade de questões respondidas com "quase sempre" ou "sempre", apresentou mínimo de zero e máxima de 11, e média na amostra de 1,78 $\pm 2,27$.

Ao se considerar a frequência das respostas "quase sempre" e "sempre" na aplicação do OHIP-14, observou-se que as dimensões de maior impacto na qualidade de vida das pessoas entrevistadas foram: desconforto psicológico, cujas perguntas se referem à preocupação $\mathrm{e}$ estresse pela condição bucal; dor física, onde pergunta-se sobre a sensação de dor e incômodo para comer; e incapacidade psicológica, relacionada à dificuldade para relaxar e ao sentimento de vergonha em função da condição bucal (Figura 4).

Quando essas respostas foram avaliadas segundo a perda dentária, pode se constatar que o percentual de indivíduos com alta perda dentária $(63,1 \%)$ foi maior no grupo que relatou impacto na dimensão "incapacidade física", cujas respostas se referem ao prejuízo na alimentação e a necessidade de ter de parar de se alimentar (Figura 4). A associação entre essas duas variáveis foi positiva com razão de prevalência de 1,50 (IC 95\%: 1,18$1,90)$, estatisticamente significante $(p=0,003)$. Para as demais dimensões, as medidas de associação não foram estatisticamente significantes (dados não apresentados).

Figura 4 - Distribuição (\%) do impacto da saúde bucal por dimensões específicas na amostra geral e segundo perda dentária. Feira de Santana, Bahia, Brasil. $(n=306)$.

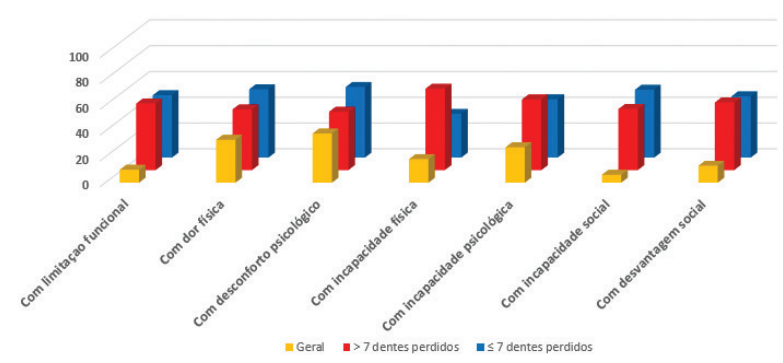

Fonte: Autoria própria

\section{DISCUSSÃO}

Os achados desse trabalho mostram que a perda dentária tem impacto negativo na qualidade de vida dos indivíduos no que se refere apenas à dimensão do OHIP-14 "incapacidade física". A associação encontrada mostra que o impacto negativo da perda dentária relacionado ao prejuízo na alimentação e à necessidade de interrompê-la foi 50\% maior (RP: 1,50; IC95\%: 1,18$1,90)$ entre os indivíduos com perda dentária maior do 
que 7 dentes do que entre aqueles com até 7 unidades dentárias perdidas.

Resultados semelhantes foram encontrados por Silva, et al. ${ }^{16}$ e Silva, et al. ${ }^{17}$, mostraram haver um grande impacto entre a quantidade de dentes perdidos e a dificuldade de realização das atividades diárias dos indivíduos, sendo que $76 \%$ dessas pessoas relataram sentir dificuldade de mastigar e de sentir o gosto dos alimentos. Outros trabaIhos também ratificam esse prejuízo na mastigação entre aqueles indivíduos com uma maior perda dentária ${ }^{18-20}$.

Embora a prevalência do impacto na saúde bucal tenha sido alta na amostra como um todo, ou seja, 60,5 \% dos participantes responderam "sempre" ou "quase sempre" para um dos itens do OHIP-14, não se observou associação da perda dentária com a prevalência do impacto. Estudos como o de Batista et al. ${ }^{21}$ e Lawrence et al. ${ }^{22}$. mostraram essa associação. Ressalta-se que em Batista et al. ${ }^{21}$, a dicotomização do impacto foi menos conservadora do que o aqui adotado, incluindo no grupo os indivíduos com respostas de "quase nunca" até "sempre".

Nesse estudo a perda dentária não representou obstáculo à interação social, diferentemente do estudo de Sanadhya et al. ${ }^{23}$ que reportou impacto da perda dentária na dimensão desvantagem social. Essas diferenças nas percepções da qualidade de vida podem ser resultados da influência cultural ${ }^{24}$.

Analisando a prevalência de perda dentária na amostra do presente estudo, constata-se que o percentual de dentes perdidos (49,05\%) foi alto na população, não diferindo muito de algumas pesquisas no Brasil. Dados do Levantamento Epidemiológico de Saúde Bucal ${ }^{4}$ mostram que o componente dente perdido do índice CPOD foi responsável por $44,7 \%$ do índice no grupo de 35 a 44 anos e $92 \%$ no grupo de 65 a 74 anos. Esse perfil de morbidade dental reforça a discussão sobre a ainda persistente prática mutiladora na população brasileira que acaba por criar uma outra demanda crítica a ser solucionada pelas autoridades responsáveis - a necessidade de reposição dentária com uso de próteses. Ressalta-se ainda que entre os indivíduos com maior perda dentária, houve pior condição bucal no que se refere à maiores médias de perda de inserção clínica periodontal, biofilme dental e do índice CPO-D, e menor número de dentes tratados. Esse quadro observado reafirma a presença de fatores determinantes para essa perda dentária como a forma de prestação dos cuidados odontológicos, acessibilidade e utilização de serviços odontológicos ${ }^{25,26}$.

Ainda com relação à análise da perda dentária nos participantes desse estudo, observou-se que a maior perda dentária foi estatisticamente associada à idade (quanto maior a faixa etária, maior a probabilidade de apresentar perdas dentárias), ao desemprego, ao menor nível de instrução, menor poder aquisitivo, e maior prole de filhos. Esses achados são consistentes com trabalhos que avaliaram os fatores associados a perda dentária em populações de adultos ${ }^{25,27,28}$, o que nos leva a compreensão de que as causas determinantes desse agravo bucal vão além das condições biológicas favoráveis à cárie ou doença periodontal e de que, assim, aspectos contextuais do indivíduo devem ser avaliados para maior controle da sua ocorrência na população.

A hipertensão foi a única morbidade sistêmica estudada que se associou a perda dentária, corroborando os achados de alguns estudos que avaliaram essa associação ${ }^{29,30}$. Alguns autores colocam que a doença periodontal, causa importante de perdas dentárias, é uma doença inflamatória que pode levar à aterosclerose subclínica e disfunção endotelial por meio da elevação de mediadores inflamatórios com implicações no controle da pressão arterial e no desenvolvimento de lesões em órgãos-alvo ${ }^{31}$. Destaca-se que esses mecanismos ainda são especulativos o quer requer estudos confirmatórios nessa linha.

Afora esses achados observados, algumas limitações necessitam ser apontadas neste trabalho. Em primeiro lugar, alguns estudos que avaliaram a perda dentária têm pontuado que a posição e distribuição dos dentes perdidos nas arcadas dentárias podem interferir nessa percepção da qualidade de vida ${ }^{32}$. No presente estudo, esses aspectos não foram considerados.

Uma segunda limitação se refere ao fato deste trabaIho apresentar dados preliminares a um estudo maior em andamento sobre qualidade de vida, requerendo empoderamento da amostra para detecção de possíveis diferenças em nível de $5 \%$ que podem não ter sido encontradas em razão do número da amostra. Em terceiro lugar, o desenho do estudo adotado impossibilita estabelecer a precedência temporal e amplia as chances de vieses de memória. Por último, chama-se atenção para a não representatividade da amostra para universo de indivíduos que frequentam o serviço público, uma vez que a seleção da amostra compreendeu indivíduos segundo alguns critérios de exclusão e apenas aqueles que buscaram o serviço por alguma razão, não abrangendo os indivíduos que não acessam a rede de serviço por algum motivo. Portanto, os resultados apresentados devem ser interpretados cuidadosamente previamente a qualquer generalização.

\section{CONCLUSÃO}

Houve associação entre alta perda dentária ( $\geq 7$ dentes) e a dimensão "incapacidade física" do OHIP-14, revelando o impacto negativo da perda dentária em dimensões da qualidade de vida que prejudicam o desempenho de atividades cotidianas e necessárias.

Esses achados são relevantes para a saúde pública e devem ser levados em consideração no planejamento local das ações de saúde bucal para populações com idade mínima de 18 anos, especialmente no que se refere a demanda para tratamento reabilitador com prótese dentária.

\section{REFERÊNCIAS}

1. KASSEBAUM, N.J. et al. Global burden of severe tooth loss: a systematic review and meta-analysis. J. dent. res., Washington, v. 93, n.7, p. 20S-28S, 2014. 
2. GERRITSEN, A.E. et al. Tooth loss and oral health-related quality of life: a systematic review and meta-analysis. Health qual. life outcomes, London, v.8, n. 126, p. 1-11, 2010

3. MARCENES, W. et al. Global Burden of Oral Conditions in 1990-2010: A systematic Analysis. J. dent. res., Washington, v. 92, n.7, p.592-597, 2013.

4. BRASIL. Ministério da saúde. SB Brasil 2010 - Pesquisa Nacional de Saúde Bucal. Resultados Principais. Brasília: Departamento de Atenção Básica, Secretaria de Atenção Básica, Ministério da Saúde, 2011.

5. WORLD HEALTH ORGANIZATION (WHO). Oral Health Surveys Basic Methods. Geneva: WHO, 1997.

6. VARGAS, A.M.D.; PAIXÃO, H.H. Perda dentária e seu significado na qualidade de vida de adultos usuários de serviço público de saúde bucal do Centro de Saúde Boa Vista, em Belo Horizonte. Ciênc. Saúde Colet., Rio de Janeiro, v. 10, n.4, p.1015-1024, 2005.

7. BATISTA, M. J. O Impacto da perda dentária na qualidade de vida de adultos. Piracicaba: UNICAMP, 2013.

8. GABARDO, M. C. L. et al. Autopercepção de saúde bucal conforme o Perfil de Impacto da Saúde Bucal (OHIP) e fatores associados: revisão sistemática. Rev. panam salud pública, Washington, v. 33, n. 6, p.439445, 2013.

9. CARVALHO, J. C. et al. Do oral health conditions adversely impact young adults? Caries res., Basel, v. 49, n. 3, p. 266-274, 2015.

10. MENDEZ, M.; GOMES, S.C. Qualidade de vida relacionada à saúde bucal: Perspectivas para a periodontia. SOBRAPE, Belo Horizonte, v.23, n.4, p. 38-44, 2013.

11. PAPANOU, P.N.; WENNSTÖM, J.L. The angular bony defect as indicator of further alveolar bone loss. J. clin. periodontol., Copenhagen, v.18, n.5, p.317-322, 1991.

12. RAMFJORD, S. Indices for prevalence and incidence of periodontal disease. J. periodontol., Indianapolis, v. 30, n.1, p.51-59, 1958.

13. LOPEZ, N. et al. Periodontal therapy may reduce the risk of preterm low birth weight in women with periodontal disease: a randomized controlled trial. J. periodontol., Indianapolis, v. 73, n. 8, p.911-924, 2002.

14. AINAMO, J.; BAY, I. Problems and proposals for recording gingivitis and plaque. Int. dent. j., London, v. 25, p. 229-235, 1975.

15. OLIVEIRA, B. H.; NADANOVSKY, P. Psychometric properties of the Brazilian version of the Oral Health Impact Profile - short form. Community dent. oral epidemiol., Copenhagen, v. 33, n. 4, p. 307-314, 2005.

16. SILVA, P. V. et al. Impacto do número de dentes no desempenho de atividades diárias. RFO, Passos Fundo, v.12, n. 3, p.13-17, 2007.

17. SILVA, M. E. S. et al. Impacto da perda dentária na qualidade de vida. Ciência Saúde Colet., Rio de Janeiro, v.15, n.3, p. 841-850, 2010.
18. BORTOLUZZI, M. C. et al. Tooth loss, chewing ability and quality of life. Contemp Clin Dent., India, v.3, n. 4. p. 393-397, 2012.

19. KHALIFA, N. et al. Chewing ability and associated factors in a Sudanese population. J oral sci., Tokyo, v. 55, n. 4, p. 349-357, 2013.

20. MOTALLEBNEJAD, M. et al. Validity and reliability of the Persian version of the oral health impact profile (OHIP)-14. Caspian J. Intern. Med., Iran, v. 2, n. 4, p. 314-320, 2011.

21. BATISTA, M. J. et al. The impacts of oral health on quality of life in working adults. Braz oral res., Sao Paulo, v. 28, n.1, p.1-6, 2014.

22. LAWRENCE, H.P. et al Oral health-related quality of life in a birth cohort of 32-year olds. Community dent. oral epidemiol., Copenhagen, v. 36, n. 4, p. 305-316, 2008.

23. SANADHYA, S. et al. Assessment and comparison of clinical dental status and its impact on oral health-related quality of life among rural and urban adults of Udaipur, India: A cross-sectional study. J. Basic Clin. Pharm., India, v. 6, n.2, p. 50-58, 2015.

24. SEIDL, E.M.F.; ZANNON, C.M.LC. Quality of life and health: conceptual and methodological issues. Cad. Saúde Pública, Rio de Janeiro, v. 20, n.2, 580-588, 2004.

25. BARBATO, P. R. et al. Perdas dentárias e fatores sociais, demográficos e de serviços associado em adultos brasileiros: uma análise dos dados do Estudo Epidemiológico Nacional (Projeto SB Brasil 2002-2003). Cad. Saude Pública, Rio de Janeiro, v. 23, n. 8, p. 1803-1814, 2007.

26. BARROS, A. J. D.; BERTOLDI, A.D. Desigualdades na utilização e no acesso a serviços odontológicos: uma avaliação em nível nacional. Ciênc. Saúde Colet., Rio de Janeiro, v. 7, p. 709-717, 2002.

27. SANTILLO, P. M. H. et al. Fatores associados às perdas dentárias entre adultos em áreas rurais do estado de Pernambuco, Brasil. Ciênc. Saúde Colet., Rio de Janeiro, v. 19, n. 2, p.581-590, 2014.

28. SILVA, D. D. et al. Fatores associados à presença de dentes em adultos de São Paulo, Brasil. Cad. Saude Pública, Rio de Janeiroo, v. 25, n.11, p. 2407-2418, 2009.

29. DARNAUD, C. et al. Oral Health and Blood Pressure: the IPC Cohort. Am. j. hypertens., New York, v. 28, n.10, p.1257-1261, 2015.

30. PERES, M. A. et al. Tooth loss is associated with increased blood pressure in adults - a multidisciplinary population-based study. J. clin. periodontol., Copehagen, v. 39, n. 9, p.824-833, 2012.

31. SCANNAPIECO, F.A. et al. Association between periodontal disiese and risk for atherosclerosis, cardiovascular disease, and stroke. A systematic review. Ann. periodontol., Chicago, v. 8, n. 1, p. 38-53, 2003.

32. ZHANG, Q. et al. Functional dental status and oral health-related quality of life in an over 40 years old chinese population. Clin. Oral Invest., Berlin, n.17, p. 1471-1480, 2013.
Submetido em: 19/12/2017

Aceito em: 16/04/2018 